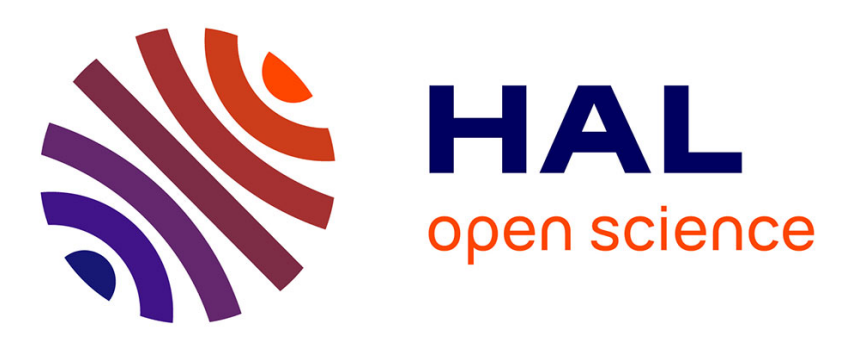

\title{
A low-cost room-level indoor localization system with easy setup for medical applications
}

Thomas Tegou, Ilias Kalamaras, Konstantinos Votis, Dimitrios Tzovaras

\section{To cite this version:}

Thomas Tegou, Ilias Kalamaras, Konstantinos Votis, Dimitrios Tzovaras. A low-cost room-level indoor localization system with easy setup for medical applications. 11th IFIP Wireless and Mobile Networking Conference (WMNC 2018), Sep 2018, Prague, Czech Republic. pp.46-52. hal-01995421

\section{HAL Id: hal-01995421 \\ https://hal.inria.fr/hal-01995421}

Submitted on 26 Jan 2019

HAL is a multi-disciplinary open access archive for the deposit and dissemination of scientific research documents, whether they are published or not. The documents may come from teaching and research institutions in France or abroad, or from public or private research centers.
L'archive ouverte pluridisciplinaire HAL, est destinée au dépôt et à la diffusion de documents scientifiques de niveau recherche, publiés ou non, émanant des établissements d'enseignement et de recherche français ou étrangers, des laboratoires publics ou privés.

\section{(c)(1)}

Distributed under a Creative Commons Attribution| 4.0 International License 


\title{
A low-cost room-level indoor localization system with easy setup for medical applications
}

\author{
Thomas Tegou*, Ilias Kalamaras*, Konstantinos Votis* and Dimitrios Tzovaras* \\ *Information Technologies Institute \\ Centre for Research and Technology Hellas \\ Thessaloniki, Greece \\ Email: \{thomtego, kalamar, kvotis, Dimitrios.Tzovaras\}@iti.gr
}

\begin{abstract}
Indoor localization systems have already wide applications for providing localized information and directions. They can also be very useful in medical applications, as an individual's indoor movements can be indicative of his/her clinical status. Research for indoor localization systems is ongoing and there are numerous solutions with different hardware or algorithmic approaches, for different use cases. Ease of setup and cost are significant factors when using indoor localization systems for medical applications. This paper proposes an indoor localization system with room-level accuracy, focusing on an easy installation procedure that can be easily followed from non-technical staff, as well as on low cost, so that it can be widely adopted. The proposed system has been mainly designed for behavioural monitoring of adults in an indoor environment. The system operates by tracking a carried device based on the processing of RSS (Received Signal Strength) measurements from Bluetooth Low Energy beacons placed in the area. The proposed system has been evaluated in realistic settings and and achieves an accuracy of $95.31 \%$ in room estimation.
\end{abstract}

Index Terms-Indoor Localization, beacons, Bluetooth Low Energy, room-level accuracy.

\section{INTRODUCTION}

In outdoor environments, GPS already provides a satisfactory localization solution, but it cannot be used in indoor environments. Due to the signal attenuation caused by the construction materials of buildings, indoor positioning systems cannot rely on this technology and as a result, have not been equally successful. Indoor positioning systems are already very useful in asset tracking, providing position-related information (e.g. in museums), providing directions (e.g. in airports), and in commercial and advertising applications (e.g. showing advertisements depending on the user's position in a store). Indoor positioning systems can also be very helpful in medical applications, for monitoring the movements of individuals when performing activities of daily living (ADL), thus extracting patterns that could infer the clinical status of the individual. In such applications, identifying logical areas of users, such as rooms and corridors, is more meaningful than providing precise locations specified by coordinate information. Our purpose was to develop an indoor localization system mainly for medical purposes, focused on easy installation and usage by individuals, in order to be used for older people monitoring.

ISBN 978-3-903176-03-4(C) 2018 IFIP
There are several commercial solutions for indoor localization, presented in more detail in section II, which are often costly and complex to install and their main application is for commercial purposes and not for medical. This paper proposes an indoor localization system with room-level accuracy and easy setup, utilizing cloud services, suitable for applications where collecting data from multiple users is necessary. The proposed system uses a tracking device carried by an individual and Bluetooth beacons positioned around the area. Our aim was to develop a low-cost room-level accuracy system for indoor localization with easy setup in order to collect data about indoor localization habits. The easy setup procedure refers to the idea that non-technical staff such as nurses can install the system by executing a short set of simple instructions, without needing additional information, such as the floorplan of the house or the exact positions of the beacons or even distance measurements.

The rest of the paper is organized as follows: Section II includes the related work, Section III includes our proposed method including the architecture of the system, Section IV includes a detailed description of the setup procedure and the description of the localization method .Finally section $\mathrm{V}$ includes an evaluation of the system regarding its accuracy. This section includes the results in room estimation in multiple positions in the floorplan of a house and details about utilizing the system in pilot studies. Finally the conclusion presents a summary and directions/considerations for future work.

\section{RELATED WORK}

There are several choices of hardware for indoor localization including Bluetooth, WLAN, RFID, UWB (Ultra-Wide Band), and ultrasound. The majority of them uses RF or audio signal characteristics, such as signal strength or TOF (Time Of Flight) measurements. These systems in most cases can provide precise position estimation with meter-level accuracy. Their disadvantages usually are their cost, their complex setup procedure, their high-power consumption, and the difficult integration. A comparative analysis can be found in [1] and [2].

Most of the existing commercial solutions, using beacons and smartphones, need the exact floorplan of the indoor environment [3] and a large number of beacons increasing 
the total cost. They are used mainly in commercial application such as shopping centers, museums or large public buildings and their main purpose is to offer information and advertisements to smartphone users depending on their positions. Some offered commercial systems utilizing beacons and smartphones are Estimote ${ }^{1}$, Indoo.rs ${ }^{2}$, Senion $^{3}$, Bluecats ${ }^{4}$. Existing applications usually insist on increasing the accuracy of coordinates estimation in LOS (Line Of Sight) environments using a large number of beacons [3], [4] and large training datasets. The following subsections present shortly the most common methods used in localization systems.

\section{A. Trilateration}

In trilateration, the distance from the source to the receiver is used to estimate the location of the user. Trilateration is a way of determining a location by measuring the distance from a mobile device to three beacons. For trilateration, at least three beacons are necessary. Measuring the RSS value from each beacon, a specific circular range is created represented as a circle with radius $r$. The current location of the user is where these circles overlap (Fig. 1), and is computed by solving a system of equations using least-squares. A detailed explanation of the method is presented in [5] and [6]. The Least SQuare (LSQ) and Weighted Least SQuare (WLSQ) techniques have been used for finding a solution that minimizes the least squares of the localization equations extracted from the calculated distances. In WLSQ, a weight is added to the system of equations to reflect the effect of shadowing over long distances. Existing work has shown that WLSQ outperforms the traditional LSQ [7]. In [6] and [8], an iterative version of trilateration is presented. Trilateration can be used when the beacons are placed in known positions, or the distances between them are known, which infers that a floorplan is available. The ROCRSSI (Ring Overlapping Based on Comparison of Received Signal Strength Indicator) method which improves trilateration multirateration localization accuracy introducing the use of circular rings with definite thickness. The position of a node is estimated using the overlapped region of these rings[9].

\section{B. Fingerprinting}

Localization based on the fingerprint method consists of two stages. The first is the offline stage where RSS measurements are collected for multiple reference points $R P(1), R P(2), \ldots, R P(N)$ in an indoor environment [10] [11]. These measurements are used in the second online stage in order to determine the position of the tracking device. The system estimates the location of an object by matching online measurements with the closest location fingerprints as seen in Fig. 2. Fingerprinting classification techniques are built upon machine learning algorithms like neural network (NN),

\footnotetext{
${ }^{1}$ https://estimote.com

${ }^{2}$ https://indoo.rs

${ }^{3}$ https://senion.com

${ }^{4}$ https://www.bluecats.com
}

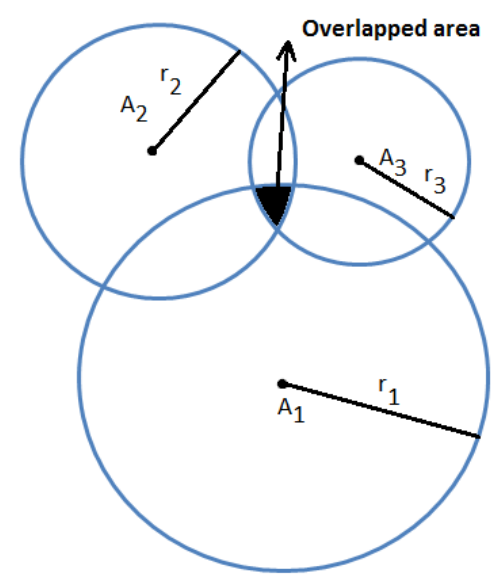

Fig. 1: A simple example of trilateration. The overlapped area defines the estimated position of the user.

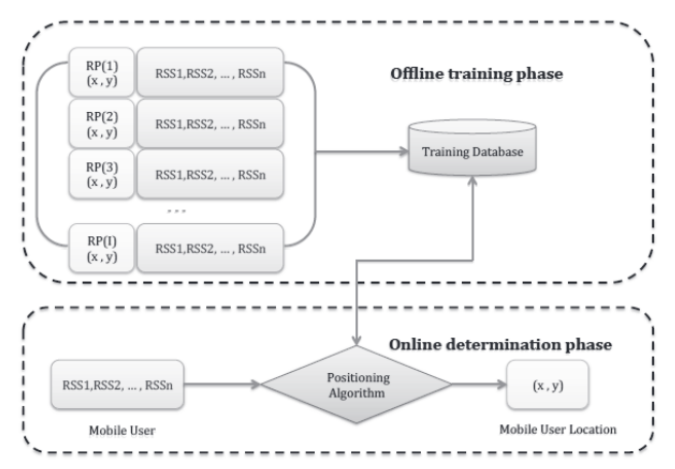

Fig. 2: A diagram of the fingerprinting method, from [3].

K-nearest neighbors (KNN), support vector machines (SVM) and Naïve Bayes (NBC). A comparison of these methods can be found in [12]. In [13], a WiFi fingerprinting indoor positioning system using single multiplicative neuron (SMN) and Principal Component Analysis (PCA), is presented, which improves accuracy and time response in location estimation.

\section{Triangulation}

Triangulation is extensively used for outdoor localization. For example, GPS uses triangulation to estimate the user's location. Triangulation locates an object by computing angles relative to multiple reference points [5]. Instead of measuring the distance directly using received signal strengths (RSS), the time of arrival (TOA) or time difference of arrival (TDOA) are usually measured, and the distance is derived by computing the attenuation of the emitted signal strength or by multiplying the radio signal velocity and the travel time. Roundtrip time of flight (RTOF) or received signal phase are also used for range estimation. UWB (Ultra Wide Band) based solutions for indoor localization utilize these methods for position estimation. A disadvantage of triangulation is that the current 
smartphones are not advanced enough to perform either of the three methods. Therefore, this method cannot currently be used to estimate the location of a smartphone without extra hardware.

\section{Hybrid Localization Methods}

Smartphones integrate many sensors such as magnetometer, accelerometer/gyroscope, ambient light sensor, etc. Multiple sensor measurements can be used in order to enhance the accuracy of position estimation. A common method to combine measurements from multiple sensors is Kalman filter. The Kalman filter is a recursive algorithm approach which takes into account past states in order to create a current estimation. [14]. In [15], the proposed system utilizes the accelerometer and the magnetometer of a smartphone to improve the position estimation accuracy. A significant improvement is observed when the trilateration method is used in combination with dead reckoning. Dead reckoning method refers to position estimation using only step detector (accelerometer) and head estimation (magnetometer).

\section{LocAlizAtion System ARchitecture}

Considering the various limitations mentioned in section II, we designed a system with a short and easy setup procedure that could be used by non-technical staff in multiple houses for medical purposes. The focus of the current paper is on developing a method that achieves a balanced trade-off between accuracy and ease of installation. Our system utilizes beacons and a tracking device, which works as a beacon scanner. This hardware is chosen due to its availability, its low cost and robustness. Using a smartphone as a tracking device, a GUI (Graphical User Interface) could be developed, facilitating the setup procedure of the beacons from nontechnical staff. A smartwatch could also be used as a tracking device. In our approach we kept a low hardware cost and a small number of beacons to be used in NLOS (Non Line Of Sight) environments.

The proposed localization system locates a tracking device (e.g. wearable smartwatch or carried smartphone) in an indoor area (e.g. a house), based on Bluetooth RSS (Received Signal Strength) measurements collected from Bluetooth beacons positioned in the area. The tracking device performs continuous Bluetooth scans for the beacon devices in order to measure their RSS. The beacons are small passive devices using Bluetooth Low Energy ${ }^{5}$. Their function is to broadcast, between small time intervals, messages containing, among others, information about their ID. These messages are scanned continuously by the tracking device, which measures the RSS received from them. The RSS is compared to a set of RSS values (fingerprints) collected during a setup phase, in order to determine the tracking device's position. The use of RSS fingerprints has the advantage that it bypasses the need of transforming the RSS values to distance which is highly dependent from the topology of each house and requires a long

\footnotetext{
${ }^{5}$ https://kontakt.io/beacon-basics/what-is-a-beacon
}

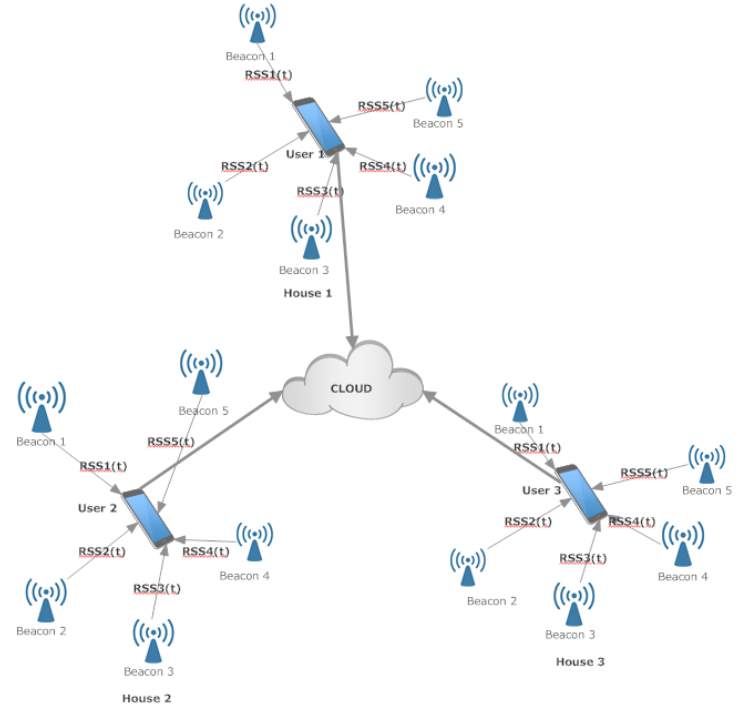

Fig. 3: An overview of the system architecture.

procedure of taking RSS measurements in various distances from the beacons.

The software components of the localization system include an application for setting up the localization installation and collecting RSS fingerprints (installed in a smartphone to be used by healthcare personnel), an application for real-time localization (installed in a smartphone or smartwatch, to be carried by the tracked person), and a cloud service used to collect the data from multiple users of the application. The main features of the system can be seen in Fig. 3 .

The idea for developing a system with exact coordinate level accuracy was abandoned because the complexity and the cost are increased. More specifically floorplans of the houses would be needed, the setup procedure would be more difficult and the cost would be higher because of the larger number of sensors. WiFi was abandoned because our purpose was to install the proposed system in multiple houses regardless of knowing the existence of a nearby deployment of routers enough to allow localization. Furthermore the use of WiFi routers instead of beacons was a more expensive solution. Finally, another advantage of beacons is their portability: they use batteries and can be placed everywhere, while WiFi routers need a power supply, limiting their possible positions in an indoor environment.

The main target of the localization system is to collect information about the indoor position of the tracked person with room-level accuracy. Collecting data for many users in a long-term basis will allow extracting patterns for activities of daily living (ADL), e.g. the time the user spends in different rooms, or whether the user spends too much time in just a single room. The main reasons that the system is based on the specific hardware choices are the low cost and the availability of the involved devices (beacons, smartphones, smartwatches). 
Moreover, using a smartphone for the installation of the setup application allows developing an interface which is necessary in order to collect training data by non-technical personnel (e.g. nurses) with a short procedure, as described in the following sections. Finally, it is quite easy for someone to wear a smartwatch or carry a smartphone in his/her pocket, used as tracking device. The reason that room-level accuracy was chosen is the simplicity of the setup procedure. A different approach with exact coordinate-level accuracy would need a much more complicated setup procedure including floorplans of the houses, distance measurements, and a larger number of beacons, increasing the total cost and the complexity.

\section{DESCRIPTION OF THE APPLICATION}

Two applications were developed for the proposed localization system: one for setup and collecting training data and one for the person who carries the tracking device. This implementation is easy to be used by non-technical staff, for example a nurse and an older person. The nurse undertakes the setup procedure, which is difficult to be done by a person with no experience of smartphones. The setup procedure is executed once for a specific house, there is no need to be repeated, unless the smartphone is used for localization in another house with a different topology and beacon positions. Having completed the training procedure, it is easy for someone to simply carry the tracking device in his/her pocket to perform localization. The setup application can run from a smartphone, because it is easy to develop a GUI, while the indoor localization application can run both from a smartphone or a smartwatch. The collected data are being synchronised with the cloud on a regular basis.

\section{A. Beacon Setup Application}

The Beacon setup application is used to initialize an instalation and collect training data. The user places the beacons in the rooms of the house and collects training data for each room. More specifically, the beacons need to be placed one for each room, in appropriate positions so that they are at least $2 \mathrm{~m}$ distant from each other, so that the collected RSS fingerprints can sufficiently discriminate the rooms. After that, he/she enters the names of the rooms were the beacons are placed, as seen in Fig. 4a. The last stage of the procedure includes the collection of training data from the RSS measurements from each room. A RSS fingerprint of each room should be created. To perform this action, the user chooses the rooms from a list in the application, as entered previously one by one and walks in every room for half a minute, as seen in Fig. 4b. An example beacon topology is presented in Fig. 5. The beacon positions are denoted with the antenna symbols. The color-shaded areas refer to representative areas inside which the person performing the installation needs to walk for half a minute, to collect fingerprints for each room.

The creation of the signal fingerprint of each room is a 30 seconds procedure. In each second, different measurements from each beacon are stored. During this short period the person performing the beacon setup is moving in the area of

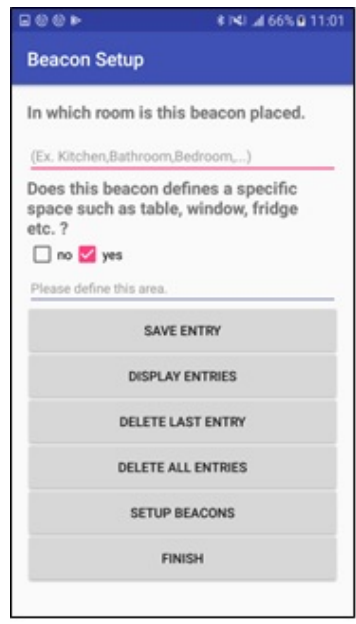

(a) A screenshot from the Beacon Setup application. The user enters the names of the rooms one by one.

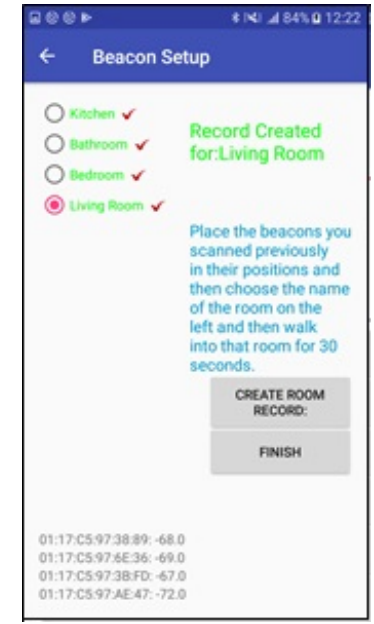

(b) A screenshot from the Beacon Setup application. On the left there is a list of the rooms. Each time the user of the application chooses a room and performs a half minute walk in the specific room.

Fig. 4: Screenshots from the setup application.

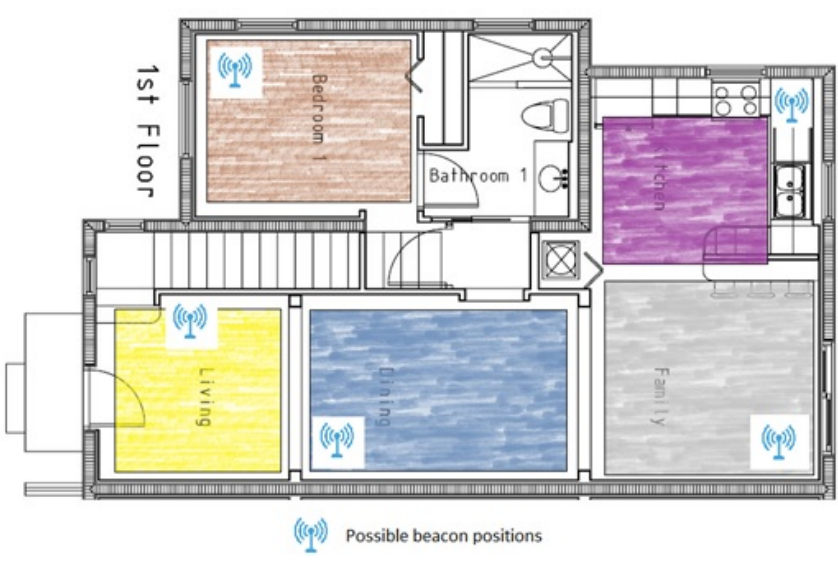

Fig. 5: An example of a beacon topology in a house. With different colors are the areas where the Beacon Setup application user should walk for half a minute in order to create the signal fingerprint for each room.

the specific room. Finally, an average is computed for each beacon representing the RSS fingerprint of the specific room. Formally, the fingerprint for a room is considered as a vector

$$
\mathbf{f}_{i}=\left(\operatorname{rss}_{i, 1}, \operatorname{rss}_{i, 2}, \ldots, \operatorname{rss}_{i, M}\right) \in \mathbb{R}^{M},
$$

where $M$ is the number of the beacons used in a house, and $\mathrm{rss}_{i, j}$ is the average from 30 measurements for beacon $j$ computed as

$$
\operatorname{rssi}_{i, j}=\frac{\sum_{k=1}^{30} r s s_{k}}{30}, k=1 \ldots 30
$$




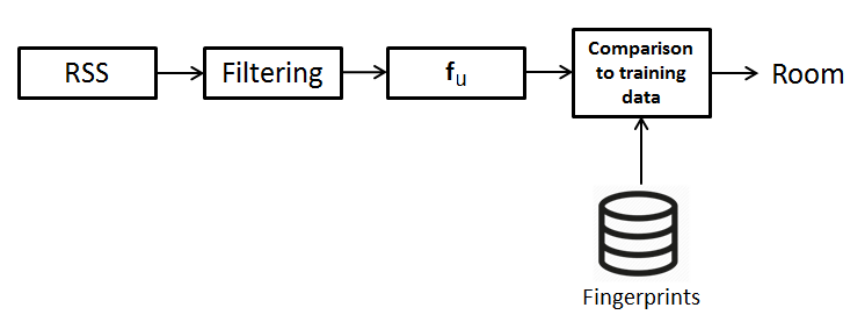

Fig. 6: Block diagram describing the room estimation procedure.

where $k$ represents the time moment. The collected fingerprints from all rooms are stored in a file, in order to later used by the indoor localization application.

\section{B. Indoor Localization Application}

After completing the setup procedure, the Indoor Localization application is able to perform localization. In general this application is based on the continuous comparison of the current RSS measurements from the beacons with the training data created by the Beacon Setup application. A block diagram of the application is presented in Fig. 6. The application stores locally in the smartphone the room change adding the timestamp of this change.

For each beacon, there is a constant length time sliding window with RSS measurements. Each time there is a new measurement from the $j$-th beacon a new value is inserted while the oldest one is discarded. The average and the standard deviation $(s)$ are computed for each time window. Values with absolute distance greater than $2 \times s$ are discarded and a final filtered average is computed. Having filtered the raw RSS measurements, a vector is formed with the filtered values. This vector is used for the comparison with the training data, the classification and the room estimation. Let

$$
\mathbf{f}_{u}=\left(\mathrm{rss}_{1}, \mathrm{rss}_{2}, \ldots, \mathrm{rss}_{M}\right) \in \mathbb{R}^{M},
$$

be the vector with the filtered RSS values. The Euclidean distance between this current fingerprint and each fingerprint of the database is computed and the minimum distance corresponds to the room where the user of the smartphone is located:

$$
\text { room }=\arg \min _{i \in \text { rooms }}\left\|\mathbf{f}_{u}-\mathbf{f}_{i}\right\|
$$

In addition, in order to decrease fluctuations between rooms, another time sliding window is created with the estimated rooms of the above procedure. Each time moment a new estimation of the room is created and stored in this sliding window while the oldest estimation is discarded as seen in Fig. 7. The appearance frequency of each room in this window is computed. Let $f r_{i}$ be the appearance frequency of room $i$ in this time window. The final room estimation is:

$$
\operatorname{room}_{\text {final }}=\arg \max \left(f r_{1}, f r_{2}, \ldots, f r_{M}\right)
$$

\begin{tabular}{|l|c|c|c|c|c|c|c|}
\hline $\mathrm{t}$ & 1 & 2 & 3 & 4 & 5 & $\ldots$ & $\mathrm{k}$ \\
\hline $\begin{array}{l}\text { Room } \\
\text { Estimation }\end{array}$ & Room1 & Room1 & Room2 & Room3 & Room1 & $\ldots$ & Room1 \\
\hline
\end{tabular}

Fig. 7: Each time moment the algorithm estimates the possible room.

when the maximum frequency is above the $80 \%$ of the length of the time window, which is 800 time time instances, then the application considers that the user is located in this room. There is also a functionality for estimating when the user is outside the monitored indoor environment. When the smartphone does not receive broadcasts from the beacons used in the training phase for a time period of 4 second the application considers that the user is outside the house.

In general, the room estimation uses the nearest neighbor of the room fingerprints. The dataset from the training phase contains equal number of elements with the rooms entered during the training phase which is usually less than 10 . Using different strategies for matching fingerprints (e.g. K-nearest neighbour) would have more meaning in applications where exact localization is estimated from large datasets where each coordinate is associated with RSS measurements.

The above applications (setup and localization) can be used to monitor the activities of an individual for a period of time, in order to extract patterns that are useful for medical purposes. Collecting localization data from several users allows for larger-scale studies, in order to study correlations of movement patterns to the clinical status of the users (e.g. frailty status). In order to collect data from multiple users from distant geographic locations, the localization application transmits the estimated room location to a central cloud repository. The data transmission can occur either automatically, when the user changes room, along with the timestamp of the change, or manually by clinical personnel, for cases of limited connectivity, by transmitting a batch of locally stored room changing records.

\section{EVALUATION OF THE PROPOSED SYSTEM}

The proposed system was tested in the KRIPIS Smart Home[16] at CERTH/ITI, Thessaloniki, Greece. The installation included five beacons in five rooms of the house. The area of the house is $8.6 \mathrm{~m} \mathrm{x} 9.35$, equal to $80.41 \mathrm{~m}^{2}$. Another additional information about the specific floorplan is that there is no wall between the kitchen and the living room. The reason for mentioning that is that a wall is a source of additional attenuation in RSS measurements [17]. The application was tested while a user was standing in multiple positions in each room, in four different directions, in order to test how the body affects the RSS and the room estimation of the application. The user remained in each position for four minutes, one minute for each direction. A smartphone was used for the test, for running both the setup and the localization applications. During the evaluation, the phone was in the user's pocket. A LG Nexus 5x smartphone and Sensoro beacons were used. 
The Sensoro beacons were chosen due to their low price and their long lasting battery (they use 4 AA batteries). Beacons using power plugs could also be used. However the majority of the beacons in the market use batteries. There are only few beacons using power plugs as power source, which are more expensive. Another disadvantage of these beacons is that they occupy power plugs probably useful for other electrical appliances.

In Fig. 8, the results of the test are presented. The total points were 48. Each circular sector corresponds to a specific orientation of the user, black color refers to a successful room estimation, while a red one to a false estimation. The test was conducted while the user was standing in four different directions in each point. The reason for testing the algorithm in multiple directions is that the human body attenuates the Bluetooth signal, as seen in [18] and [19]. If the human body interferes between a smartphone and a beacon then additional attenuation is inserted in the RSS measurements. Considering each direction as a different position the total positions are $48 \times 4=192$. The total number of wrong estimations are 12, which corresponds to a percentage of 93.75\% in succesfull room estimation. As can be seen in Fig. 8 , the wrong estimation points are located in the limits of the rooms. In all erroneous cases, the error was that the estimated room was a neighboring room to the actual room, e.g. making an estimation of "living room" when the actual room was "bedroom", or making an estimation of "kitchen" when the actual room was "living room".

Another experiment was conducted increasing the number of beacons from 5 to 8 . Two beacons were placed in the three largest rooms of the house instead of one, dividing each of these rooms into two subspaces. The training phase was conducted considering each subspace as an individual room. The results of the second testbed are presented in Fig. 9 and show a small increase in room estimation accuracy from $93.75 \%$ to $95.31 \%$ while the total number of wrong room estimations was decreased from 12 to 9.The increase in the number of beacons led to an improvement in accuracy, which was rather expected. However using more beacons the cost increases and the training phase takes more time. Both experiments were conducted in real world conditinons were other persons coexisted with the monitored one. Moreover other bluetooth and WiFi devices, which occupy the $2.4 \mathrm{GHz}$ band and create interference were in operation. Despite these sources of interference the room estimation accuracy was above $93 \%$ in both experiments.

A preliminary evaluation of the proposed system in largescale pilots has also started in the context of the FrailSafe European project [20]. The purpose of the pilot tests is the identification of factors related to frailty in older adults, based, among others, on behavioural characteristics extracted from the user indoor movements while performing activities of daily living. The system is being used in three geriatric clinical centers, in Nancy (France), Nicosia (Cyprus) and Patras (Greece), with a total of about 300 older individuals.

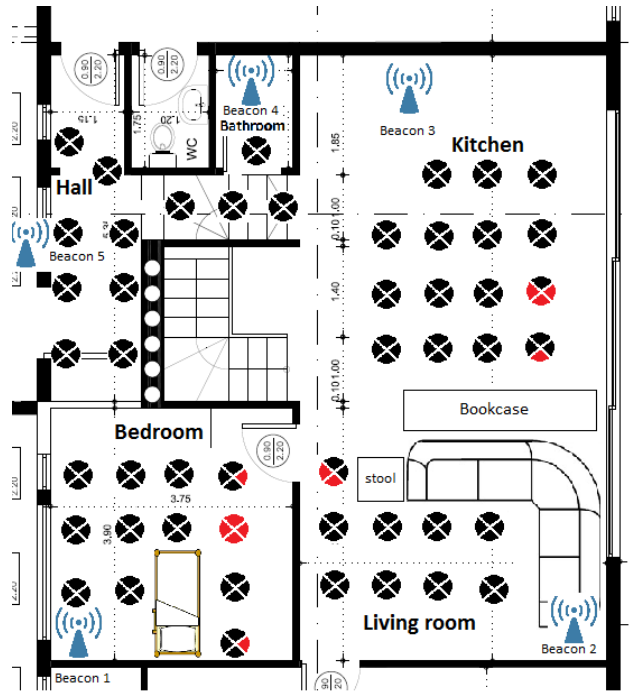

Fig. 8: The floorplan of the house where the evaluation test was conducted. Each section of the circle represents a different direction of the user. Red color represents wrong room estimations.

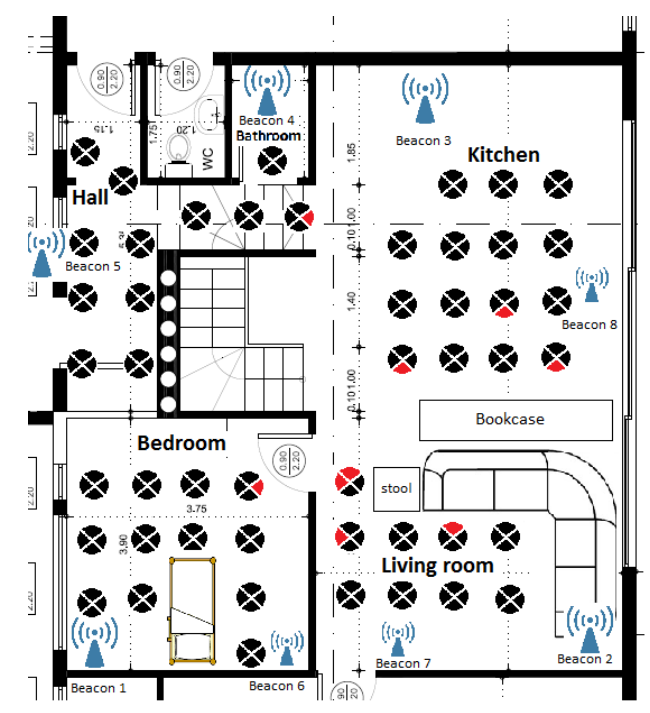

Fig. 9: The results of the second experiment where the number of beacons increased from 5 to 8 . Each section of the circle represents a different direction of the user. Red color represents wrong room estimations.

The setup procedure is performed by nurses in the participants' houses. The aim is to collect behavioral data and extract activity patterns. For instance, very long time spent at a single room may be an indication of bad psychological state, or difficulty in movement. Analyzing such patterns at a later stage can lead to possible correlations of movement patterns to frailty symptoms. 


\section{CONCLUSION}

A room-level accuracy indoor localization system was presented, focusing on easy setup and low cost. The system is based on beacons using the Bluetooth Low Energy protocol. The main features of the system are the two applications, one to be used to collect training data, in the form of RSS fingerprints, and the other one to be used for localization and for syncing data with the cloud. The evaluation in an experimental setting gives an accuracy of $93.75 \%$ in room estimation which increased to $95.31 \%$ using more beacons. The future work includes changes for achieving sub-room and exact-coordinate level accuracy and modifications for outdoor use. Although the proposed method is targetted for indoor use, it could also be extended for outdoor use. In case of outdoor use it could be useful to test the system in comparison with GPS-based applications where a greater accuracy is needed. For example GPS $^{6}$ average accuracy for smartphones is $4.9 \mathrm{~m}$. If a greater accuracy is needed other solutions using BLE and beacons would be probably more successful. Another evaluation test could be performed, by limiting sources of interference, such as other bluetooth or WiFi devices or coexisting persons in the same area with the monitored one. Hardware changes are also included in the future work: UWB (Ultra-Wide-Band) modules is an alternative choice for achieving an exact coordinate estimation. Evaluation in more real-world installations is also a direction for future work, to evaluate the applicability of the method in behavioral pattern extraction for medical purposes.

\section{ACKNOWLEDGMENT}

This work is supported by the EU funded project FrailSafe (H2020-PHC-2015-single-stage, grant agreement no. 690140).

\section{REFERENCES}

[1] L. Mainetti, L. Patrono, and I. Sergi, "A survey on indoor positioning systems," in Software, Telecommunications and Computer Networks (SoftCOM), 2014 22nd International Conference on. IEEE, 2014, pp. 111-120.

[2] Y. Gu, A. Lo, and I. Niemegeers, "A survey of indoor positioning systems for wireless personal networks," vol. 11, no. 1. IEEE, 2009, pp. 13-32.

[3] D. Čabarkapa, I. Grujić, and P. Pavlović, "Comparative analysis of the bluetooth low-energy indoor positioning systems," in Telecommunication in Modern Satellite, Cable and Broadcasting Services (TELSIKS), 2015 12th International Conference on. IEEE, 2015, pp. 76-79.

[4] https://estimote.com, accessed: 2018-04-17.

[5] P. K. Yoon, S. Zihajehzadeh, B.-S. Kang, and E. J. Park, "Adaptive kalman filter for indoor localization using bluetooth low energy and inertial measurement unit," in Engineering in Medicine and Biology Society $(E M B C), 2015$ 37th Annual International Conference of the IEEE. IEEE, 2015, pp. 825-828.

[6] A. N. Raghavan, H. Ananthapadmanaban, M. S. Sivamurugan, and B. Ravindran, "Accurate mobile robot localization in indoor environments using bluetooth," in Robotics and Automation (ICRA), 2010 IEEE International Conference on. IEEE, 2010, pp. 4391-4396.

[7] N. Lasla, A. Bachir, and M. Younis, "Area-based vs. multilateration localization: A comparative study of estimated position error," in Wireless Communications and Mobile Computing Conference (IWCMC), 2017 13th International. IEEE, 2017, pp. 1138-1143.

\footnotetext{
${ }^{6}$ https://www.gps.gov/systems/gps/performance/accuracy/
}

[8] W.-Y. Chung et al., "Enhanced rssi-based real-time user location tracking system for indoor and outdoor environments," in Convergence Information Technology, 2007. International Conference on. IEEE, 2007, pp. 1213-1218.

[9] E. Cassano, F. Florio, F. De Rango, and S. Marano, "A performance comparison between roc-rssi and trilateration localization techniques for wpan sensor networks in a real outdoor testbed." in Wireless Telecommunications Symposium, 2009. WTS 2009. IEEE, 2009, pp. $1-8$.

[10] A. M. Hossain, H. N. Van, and W.-S. Soh, "Fingerprint-based location estimation with virtual access points," in Computer Communications and Networks, 2008. ICCCN'08. Proceedings of 17th International Conference on. IEEE, 2008, pp. 1-6.

[11] P. Lin, Q. Li, Q. Fan, X. Gao, and S. Hu, "A real-time locationbased services system using wifi fingerprinting algorithm for safety risk assessment of workers in tunnels," Mathematical Problems in Engineering, vol. 2014, 2014.

[12] L. B. Del Mundo, R. L. D. Ansay, C. A. M. Festin, and R. M. Ocampo, "A comparison of wireless fidelity (wi-fi) fingerprinting techniques," in ICT Convergence (ICTC), 2011 International Conference on. IEEE, 2011, pp. 20-25.

[13] Y. Basiouny, M. Arafa, and A. M. Sarhan, "Enhancing wi-fi fingerprinting for indoor positioning system using single multiplicative neuron and pca algorithm," in Computer Engineering and Systems (ICCES), 2017 12th International Conference on. IEEE, 2017, pp. 295-305.

[14] C. Langlois, S. Tiku, and S. Pasricha, "Indoor localization with smartphones," to appear, IEEE Consumer Electronics, 2017.

[15] J. Röbesaat, P. Zhang, M. Abdelaal, and O. Theel, "An improved ble indoor localization with kalman-based fusion: An experimental study," Sensors, vol. 17, no. 5, p. 951, 2017.

[16] CERTH/ITI Smart Home, https://smarthome.iti.gr/.

[17] T. van Dijk, Indoor localization using BLE. Using Bluetooth Low Energy for room-level localization. Faculty of Mechanical, Maritime and Materials Engineering (3mE) Delft University of Technology, 2016.

[18] G. Yan, N. Che, H. Liu, and Y. Tang, "How to confirm ibeacon direction?" in Electronic Engineering and Information Science: Proceedings of the International Conference of Electronic Engineering and Information Science 2015 (ICEEIS 2015), January 17-18, 2015, Harbin, China. CRC Press, 2015, p. 139.

[19] S. Sen, R. R. Choudhury, and S. Nelakuditi, "Spinloc: Spin once to know your location," in Proceedings of the Twelfth Workshop on Mobile Computing Systems \& Applications. ACM, 2012, p. 12.

[20] "European Project FrailSafe," http://frailsafe-project.eu/, accessed: 2018-03-30 